\title{
ON THE DYNAMICS OF THE RbI CRYSTAL.
}

\author{
BY T. A. HOFFMANN. \\ (RECEIVED SEPTEMBER 15, 1946.)
}

The lattice constant, the compressibility and the infra-red wave-lenght of the $R b I$ crystal are calculated by a statistical atomic model improved by Gombás. The results show, that this model which includes the correlation effect is much more adaptable to problems, where the outer parts of the atoms or ions are of the greatest importance. Estimations are made for the lattice energy and for the ideal tensile strength of the $R b I$ crystal on the same basis. All these calculations are made without any empirical or semi-empirical parameter.

\section{1. §. INTRODUCTION.}

Among the various types of solids the ionic crystals can be dealt with the most easily, for their bond is given, in rough approximation, by the electrostatic forces between the ions. Of course there must be certain repelling forces also besides the Coulomb-forces, in order to make possible a stable position at an equilibrium spacing, where the two forces compensate each other. The first attempts took these repelling forces semi-empirically, ${ }^{1}$ for instance, as $A / r^{n}$, or $B e^{-r / r_{0}}$, where $r$ is the distance of the two ions and $A, B, n, r_{0}$ are constants determined by experiments.

Wave mechanics can interpret these repelling forces, but the calculations are, especially for systems containing many "electrons, rather lengthy, or need a great mechanical computating apparatus. Therefore these calculations were performed only for crystals built up from ions on the top of the periodic chart: $\mathrm{LiH}^{2}{ }^{3}, \mathrm{LiF}^{3}, \mathrm{NaCl}^{4}$.

The problem can be dealt with more easily by the statistical atomic model. This model gives the best approximations in the case of noblegas atoms or noble-gas-like ions for their spherical symmetry. This circumstance will make possible the investigation of the alkali halogenides. The model, being a statistical one, favours evidently systems containing

${ }^{1}$ See, for instance, the article of M. Born and M. Göppert-Mayer in the Handbuch der Physik XXIV/2. Second edition, p. 704 and seq.

2 E. A. Hylleraas, Zeitschr. f. Phys. 63, p. 771 (1930).

3 D. Ewing and F. Seitz, Phys. Rev. 50, p. 760 (1936).

- R. Landshoff, Zeitschr. f. Phys. 102, p. 201 (1936); W. Shockley, Phys. Rev. 50, p. 754 (1936). 
many electrons. That's why we took the $R b I$ crystal as the subject of our investigations.

Jensen ${ }^{5}$ has performed calculations for the whole alkali halogenide series by the non-corrected statistical model, further Neugebauer and Gombás $^{6}$ for $K C l$ and Gombás ${ }^{7}$ for $\mathrm{LiBr}$, taking into account the electrostatic polarisation.

Jensen has made his calculations by the combination of the ThomasFermi-Dirac and the Fermi-Amaldi models. The Gombás model which takes into account the correlation effect improves the models essentially in the outer parts of the atoms or ions. Computing the interaction of ions in crystals, just these outer parts are of the greatest importance and so it is not without interest to calculate the dynamical constants on the basis of the Gombás model. ${ }^{8}$

\section{2. §. APPROXIMATION OF THE ELECTRON DENSITIES BY ANALYTICAL FUNCTIONS.}

We do not perform the whole calculation starting from the Gombás model, but we use a perturbation method, taking as zero-order results those of Jensen. First of all we make an approximation for the electron density distributions of $I^{-}$and $R b^{+}$by analytical functions. The interactions of the electron clouds in the crystal lattice result from the double or multiple overlapping of the electron clouds. Thus the integrals occurring at the calculations are to be extended to these overlapping regions only, the outer parts of the ions, and in these parts the densities can be approximated well by analytical functions.

Jensen uses for the density distribution

$$
\nu(r)=\nu(\mu x)=\frac{Z N}{4 \pi \mu^{3}(N-1)}\left(\frac{\psi_{0}+k \eta}{x}\right)^{3 / 2}
$$

where $Z$ is the atomic number, $N$ the number of electrons of the ion,

$$
\mu=\left(\frac{9 \pi^{2}}{2}\right)^{1 / 3} \frac{1}{4 Z^{1 / 3}}\left(\frac{N}{N-1}\right)^{2 / 3} a_{H}
$$

$\psi_{0}$ is the solution of the Thomas-Fermi differential equation and $\eta$ the

${ }^{5} H$. Jensen, Zeitschr. f. Phys. 77, p. 772 (1932); 101, p. 164 (1936). This last article will be cited in the following shortly as "Jensen"4.

- T. Neugebauer and P. Gombás, Zeitschr. f. Phys. 89, p. 480 (1934).

7 P. Gombás, Zeitschr. f. Phys. 92, p. 796 (1934).

8 P. Gombás, Zeitschr. f. Phys. 121, p. 523 (1943). 
correction function for ions, both tabulated functions, and finally $k$ a constant computable from the boundary conditions for each ion separately. ${ }^{10}$

There will be convenient to make an approximation for $\nu$ in the region used in the form

$$
\nu(r)=C \frac{e^{\alpha r}}{r^{3 n}},
$$

where $n$ is an integer. The choice $3 \pi$ of the exponent in the denominator is convenient, because thus even $\nu^{1 / 3}$ has an integer exponent in the denominator, and the occurring integrations can be made in closed forms.

In the following we shall use atomic units throughout, except we state the contrary explicitely. For $I^{-}$we have

$$
\begin{array}{ll}
Z_{1}=53, \quad N_{1}=54, & \mu_{1}=0,23864, \quad k_{1}=1,15 \cdot 10^{-6}, \\
& r_{01}=5,75,
\end{array}
$$

$r_{01}$ being the radius of the ion. With these values we find

$$
\nu_{1}=0,91851 \frac{e^{0,784 r}}{r^{6}},
$$

as a good approximation in the interval $1>r>6$.

Similarly for $R b^{+}$we have

$$
\begin{gathered}
Z_{2}=37, \quad N_{2}=36, \quad \begin{array}{c}
\mu_{2}=0,27072, \quad k_{2}=-10,7 \cdot 10^{-6} \\
r_{02}=3,65
\end{array}
\end{gathered}
$$

and

$$
\nu=2,27402 \frac{e^{-0,89802 r}}{r^{3}}
$$

for the interval $1<r<4$.

These approximations differ in the average about $5-10 \%$ from the true values of $\boldsymbol{\nu}$ and this is sufficient for our purpose.

E. Fermi, Zeitschr. f. Phys. 48, p. 73 (1928); V. Bush and S. Cáldwell, Phys. Rev. 38, p. 1898 (1931); E. B. Baker, Phys. Rev. 36, p. 630 (1931); C. Miranda, Lincei Rend. 5, p. 285 (1934); A. Sommerfeld, Zeitschr. f. Phys. 78, p. 283 (1932).

${ }_{10}$ H. Jensen, Zeitschr. f. Phys. 101, p. 141 (1936). 
The electron densities of Gombás could be calculated in a similar way using instead of Jensen's values for $k$ those of Gombás. ${ }^{11}$ Instead of this $w \geq$ start from the electron densities of Jensen. The correction is the difference of $\nu^{\prime}$ and $\nu$ ( $\nu^{\prime}$ being Gombás's electron density), which is in the first approximation

$$
\begin{aligned}
\Delta \nu=\nu^{\prime}-1 & =\frac{Z N}{4 \pi \mu^{3}(N-1)}\left[\left(\frac{\psi_{0}+k^{\prime} \eta}{x}\right)^{3 / 2}-\left(\frac{\psi_{0}+k \eta}{x}\right)^{3 / 2}\right]= \\
& =\frac{3 Z N}{8 \pi \mu^{3}(N-1)}\left(k^{\prime}-k\right) \frac{\psi_{0}^{1 / 2} \eta}{x^{3 / 2}} .
\end{aligned}
$$

In the interval $4<x<25$, where our calculations are made, $\frac{\psi_{0}^{1} / 2 \eta}{x^{3 / 2}}$ can be taken in an excellent approximation as a linear function of $x$, especially

$$
\frac{\psi_{0}^{1 / 2 \eta}}{x^{3 / 2}}=0,07477 x+0,31138
$$

This approximation has a mean error of $5 \%$ in the above interval. Gombás gives ${ }^{12}$

$$
k_{1}^{\prime}=1,82 \cdot 10^{-6} \text { and } k_{2}^{\prime}=-9,8 \cdot 10^{-6} .
$$

So we get the following corrections for the electron densities

$$
\Delta \nu_{1}=9,9565 \cdot 11^{-5} r+9,8949 \cdot 10^{-5},
$$

being valid for $1<r<6$, and

$$
\Delta \nu_{2}=5,6917 \cdot 10^{-5} r+6,4167 \cdot 10^{-5},
$$

in the interval $1<r<4$.

We have further in the Gombás model

$$
r_{01}^{\prime}=5,32 \text { and } r_{02}^{\prime}=3,55
$$

for $I^{-}$and $R b^{+}$respectively.

11 See ref. 8.

12 See ref. 8. 


\section{3. §. CALCULATION OF THE LATTICE CONSTANT.}

Now we reproduce briefly Jensen's calculations. The $R b I$ crystal has a face-centred cubic lattice of the $\mathrm{NaCl}$ type. The lattice constant bsing $\delta$, the distance of two neighbouring ions of opposite signs is $\delta$ and that of two neighbouring ions of the same sign is $\sqrt{2} \delta$. Each ion has 6 neighbours of the opposite sign and 12 of the same sign. One has to calculate the lattice energy per ion pair. This is the difference between the energy of the ions in the lattice and that of the free ions per ion pair. The cause of this energy change is the electrostatic energy of the ionic charges and the change of energy, which results from the double or multiple overlapping of the electron clouds.

In the case of $R b I$ there is a double overlapping between a $I^{-}$and a neighbouring $R b^{+}$ion and one between two adjacent $I$ - ions; there is a threefold overlapping between two neighbouring $I^{-}$ions and a $R b^{+}$ion. This threefold overlapping extends only over a very small region and so it is negligible. (See figure 1.)

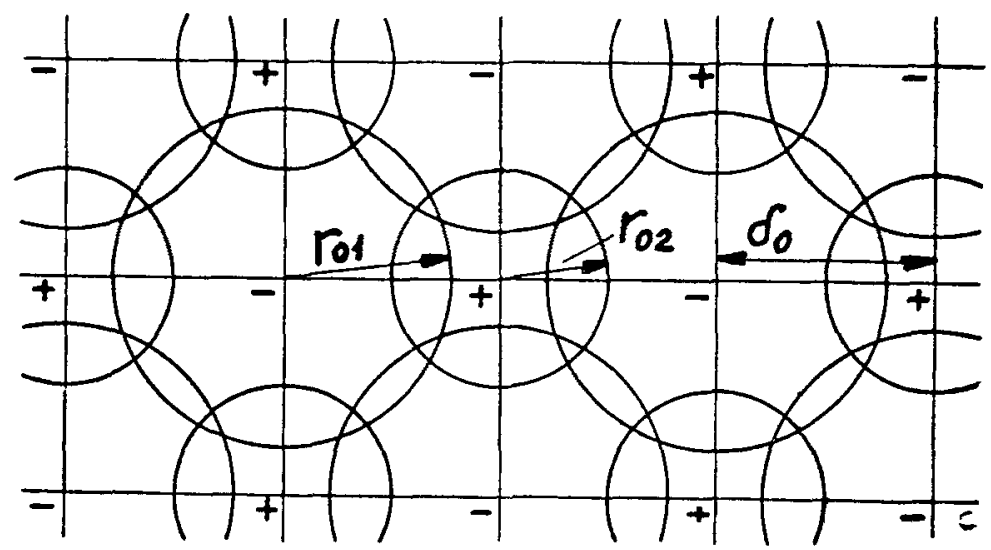

Fig. 1 .

As each $I^{-}$has 6 neighbouring $R b^{+}$ions, the double overlapping between two of them is to be taken 6 times per ion pair. Similarly, as each $I^{-}$has 12 neighbouring $I^{-}$ions, but taking the interaction with each of them each $I^{-}$is counted twice, even this interaction ought to be taken 6 times per ion pair.

The elecrostatic energy term of the ionic charges is according to Jensen

$$
E_{0}(\delta)=-\frac{1,7476}{\delta}
$$


The energy resulting from the overlapping of two ions of opposite sign is

$$
E_{1}(\delta)=6 W_{e}^{1,2}(\delta)+6 W_{a}^{1,2}(\delta)+6 W_{k}^{1,2}(\delta),
$$

and that from the overlapping of two ions of the same sign is

$$
E_{\dot{2}}(\delta)=6 W_{e}^{1,1}(\sqrt{2} \delta)+6 W_{a}^{1,1}(\sqrt{2} \delta)+6 W_{k}^{1,1}(\sqrt{2} \delta)
$$

We make the approximation, that the densities are additive in the area of the double covering. In this case, if we have an energy density term of the form $f(\nu)$ at the free ion, this term will contribute to the lattice energy a term

$$
F=\int\left[f\left(\nu_{1}+\nu_{2}\right)-f\left(\nu_{1}\right)-f\left(\nu_{2}\right)\right] d \tau
$$

where $\nu_{1}$ and $\nu_{2}$ are the electron densities of the two ions respectively and the integration is to be extended to the region of the overlapping.

So the meaning of the quantities on the right of (11) and (12) are

$$
\begin{aligned}
& W_{e}^{1,2}(\delta)=-\int \nu_{1}\left(r_{1}\right) \gamma_{2}\left(r_{2}\right) d \tau, \\
& W_{a}^{1,2}(\delta)=-x_{a} \int\left[\left(\nu_{1}\left(r_{1}\right)+\nu_{2}\left(r_{2}\right)\right)^{4 / 3}-\nu_{1}\left(r_{1}\right)^{4 / 3}-\nu_{2}\left(r_{2}\right)^{4 / 3}\right] d \tau, \\
& W_{k}^{1,2}(\delta)=\varkappa_{k} \int\left[\left(\nu_{1}\left(r_{1}\right)+\nu_{2}\left(r_{2}\right)\right)^{5 / 3}-\nu(r)^{5 / 3}-\nu_{2}\left(r_{2}\right)^{5 / 3}\right] d \tau,
\end{aligned}
$$

where $r_{1}$ and $r_{2}$ are the distances of the volume element of the integrand from the two nuclei, which are at a distance of $\delta, \nu_{1}\left(r_{1}\right)$ and $\nu_{2}\left(r_{2}\right)$ are the corresponding electron densities, and the definition of $\gamma_{2}$ is

$$
\gamma_{2}\left(r_{2}\right)=V_{e 2}\left(r_{2}\right)+\frac{N_{2}}{r_{2}}
$$

where $N_{2}$ denotes the number of electrons of the ion with the index 2 and $V_{e 2}\left(r_{2}\right)$ the electrostatic potential of the electrons of this ion at a distance $r_{2}$ from the nucleus of the same ion, further $\bar{x}_{a}$ and $x_{k}$ are constants :

$$
x_{a}=0,74553 \quad x_{k}=2,87074 .
$$

The definitions are similar, if the two ions are of the same kind. 
The whole lattice energy is the sum of (10), (11) and (12),

$$
E(\delta)=E_{0}(\delta)+E_{1}(\delta)+E_{\mathbf{2}}(\delta) .
$$

The lattice constant in the equilibrium state, is given at zero absolute temperature by the equation

$$
\frac{d E}{d \delta}=0 .
$$

Jensen's result is in this way

$$
\delta_{0}=7,48,
$$

which gives a lattice distance $8 \%$ larger, than the exprimental value $:^{13}$

$$
\delta_{\text {exp. }}=6,93 \text {. }
$$

Jensen gets a better result taking into account the van der Waals correction also. The van der Waals energy term is ${ }^{14}$

$$
E_{\text {v. d. w. }}=\frac{1394}{\delta^{6}} .
$$

Thus the lattice constant decreases to

$$
\delta_{0}=7,48 \text {, }
$$

giving a difference of $4,6 \%$ only. ${ }^{15}$ If we take into the account the van der Waals forces in this way, they have only an informative character, the constant of (19) being not exactly known.

Now we shall calculate the lattice constant by the Gombás model. The lattice energy in the corrected model is

where

$$
E^{\prime}(\delta)=E_{0}(\delta)+E_{1}^{\prime}(\delta)+E_{2}^{\prime}(\delta)=E(\delta)+\Delta E(\delta),
$$

$$
E_{1}^{\prime}(\delta)=E_{1}(\delta)+\Delta E_{1}(\delta),
$$

13 See ref. 1.

${ }_{14}$ E. J. Mayer, J. Chem. Phys. 1, p. 270 (1933).

15 There is an obvious misprint in Jensen's Tab. 4., where he gives $3,5 \%$ for the deviation. I have overtaken the same error in Phys. Rev. 70, p. 981 (1946). 


$$
E_{2}^{\prime}(\delta)=E_{2}(\delta)+\Delta E_{2}(\delta)
$$

and so

$$
\Delta E(\delta)=\Delta E_{1}(\delta)+\Delta E_{2}(\delta)
$$

We have further

$$
\begin{gathered}
\Delta E_{1}(\delta)=6\left[W_{e}^{\prime 1,2}(\delta)-W_{e}^{1,2}(\delta)\right]+6\left[W_{a}^{\prime 1,2}(\delta)-W_{a}^{1,2}(\delta)\right]+ \\
+6\left[W_{k}^{\prime 1,2}(\delta)-W_{k}^{1,2}(\delta)\right] \\
\Delta E_{2}(\delta)=6\left[W_{e}^{\prime 1,1}(\sqrt{2} \delta)-W_{e}^{1,1}(\sqrt{2} \delta)\right]+ \\
+6\left[W_{a}^{\prime 1,1}(\sqrt{2} \delta)-W_{a}^{1,1}(\sqrt{2} \delta)\right]+6\left[W_{k}^{\prime 1,1}(\sqrt{2} \delta)-W_{k}^{1,1}(\sqrt{2} \delta)\right]
\end{gathered}
$$

where

$$
\begin{gathered}
W_{e}^{\prime 1,2}(\delta)=-\int \nu_{1}^{\prime}\left(r_{1}\right) \gamma_{2}^{\prime}\left(r_{2}\right) d \tau \\
\left.W_{a}^{\prime 1,2}(\delta)=-x_{a}^{\prime} \int\left[\left(\nu_{1}^{\prime}\left(r_{1}\right)+\nu_{2}^{\prime} r_{2}\right)\right)^{4 / 3}-\nu_{1}^{\prime}\left(r_{1}\right)^{4 / 3}-\nu_{2}^{\prime}\left(r_{2}\right)^{4 / 3}\right] d \tau \\
\left.W_{k}^{\prime 1,2}, \delta=x_{k} \int\left[\left(\nu_{1}^{\prime}\left(r_{1}\right)+\nu_{2}^{\prime}, r_{2}\right)\right)^{5 / 3}-\nu_{1}^{\prime}\left(r_{1}\right)^{5 / 3}-\nu_{2}^{\prime}\left(r_{2}\right)^{5 / 3}\right] d \tau
\end{gathered}
$$

and

$$
\gamma_{2}^{\prime}\left(r_{2}\right)=V_{e 2}^{\prime}\left(r_{2}\right)+\frac{N_{2}}{r_{2}}
$$

In (26)-(28) the integrals are to be extended to regions with limiting radii $r_{01}^{\prime}$ and $r_{02}^{\prime}$ respectively, and with a nuclear distance $\delta$, whereas (13)-(15) to regions with $r_{01}, r_{02}$ and $\delta$. We have further

$$
\varkappa_{a}^{\prime}=1,13 \varkappa_{a}=0,84245 .
$$

The lattice constant, $\delta_{0}^{\prime}$, is given by the equation

$$
\frac{d E^{\prime}}{d \delta}=0
$$

Now we can take Jensen's $\delta_{0}$ as a good first approximation. So $\delta_{0}^{\prime}$ can be written in the form

$$
\delta_{0}^{\prime}=\delta_{0}+\Delta \delta_{0},
$$


where $\Delta \delta_{0}$ is so small against $\delta_{0}$ that its second or higher powers can be neglected. We can write therefore

$$
\begin{aligned}
& {\left[\frac{d E^{\prime}}{d \delta}\right]_{\delta=\delta_{0}^{\prime}}=\left[\frac{d E}{d \delta}\right]_{\delta=\delta_{0}^{\prime}}+\left[\frac{d \Delta E}{d \delta}\right]_{\delta=\delta_{0}^{\prime}}=\left[\frac{d E}{d \delta}\right]_{\delta=\delta_{0}}+} \\
& +\Delta \delta_{0}\left[\frac{d^{2} E}{d \delta^{2}}\right]_{\delta=\delta_{0}}+\left[\frac{d \Delta E}{d \delta}\right]_{\delta=\delta_{0}}+\Delta \delta_{0}\left[\frac{d^{2} \Delta E}{d \delta^{2}}\right]_{\delta=\delta_{0}}
\end{aligned}
$$

From (18), (30) and (32) we have finally

$$
\Delta \delta_{0}=-\frac{\left[\frac{d \Delta E}{d \delta}\right]_{\delta=\delta_{0}}}{\left[\frac{d^{2} E}{d \delta^{2}}\right]_{\delta=\delta_{0}}+\left[\frac{d^{2} \Delta E}{d \delta^{2}}\right]_{\delta=\delta_{0}}} .
$$

The value of $\left[\frac{d^{2} E}{d \delta^{2}}\right]_{\delta=\delta_{0}}$ can be taken, as we shall see later, from Jensen's article and so we have to calculate only $\left[\frac{d \Delta E}{d \delta}\right]_{\delta-\delta_{0}}$ and $\left[\frac{d^{2} \Delta E}{d \delta^{2}}\right]_{\delta=\delta_{0}}$.

$\Delta E_{1}$ and $\Delta E_{2}$ can be transformed to the following form:

$$
\begin{aligned}
& \Delta E_{1}=6 \int_{(1)} F_{1}\left(r_{1}, r_{2}\right) d \tau+6 \int_{(2)} F_{2}\left(r_{1}, r_{2}\right) d \tau, \\
& \Delta E_{2}=6 \int_{(3)} F_{3}\left(r_{1}, r_{2}\right) d \tau+6 \int_{(4)} F_{4}\left(r_{1}, r_{2}\right) d \tau,
\end{aligned}
$$

where the region (1) means the shaded part of figure 2, with $r_{01}, r_{02}$ and $\delta_{0}$, (2) the same with $r_{01}^{\prime}, r_{02}^{\prime}$, and $\delta_{0}$, (3) that with $r_{01}, r_{01}$ and $\sqrt{2} \delta_{0}$ and finally (4) that with $r_{01}^{\prime}, r_{01}^{\prime}$ and $\sqrt{2} \delta_{0}$. The functions $F$ are the following

$$
\begin{aligned}
F_{1}\left(r_{1} r_{2}\right)= & \nu_{1}\left(r_{1}\right) \gamma_{2}\left(r_{2}\right)+\chi_{a}\left[\left(\nu_{1}\left(r_{1}\right)+\nu_{2}\left(r_{2}\right)\right)^{4 / 3}-\nu_{1}\left(r_{1}\right)^{4 / 3}-\nu_{2}\left(r_{2}\right)^{4 / 3}\right]- \\
& -\varkappa_{k}\left[\left(\nu_{1}\left(r_{1}\right)+\nu_{2}\left(r_{2}\right)\right)^{5 / 3}-\nu_{1}\left(r_{1}\right)^{5 / 3}-\nu_{2}\left(r_{2}\right)^{5 / 3}\right], \\
F_{2}\left(r_{1}, r_{2}\right)= & -\nu_{1}^{\prime}\left(r_{1}\right) \gamma_{2}^{\prime}\left(r_{2}\right)-\chi_{a}^{\prime}\left[\left(\nu_{1}^{\prime}\left(r_{1}\right)+\nu_{2}^{\prime}\left(r_{2}\right)\right)^{4 / 3}-\nu_{1}^{\prime}\left(r_{1}\right)^{4 / 3}-\nu_{2}^{\prime}\left(r_{2}\right)^{4 / 3}\right]+
\end{aligned}
$$




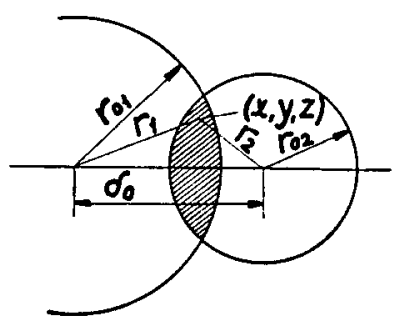

Fig. 2.

$$
\begin{aligned}
& +x_{k}\left[\left(\nu_{1}^{\prime}\left(r_{1}\right)+\nu_{2}^{\prime}\left(r_{2}\right)\right)^{5 / 3}-\nu_{1}^{\prime}\left(r_{1}\right)^{5 / 3}-\nu_{2}^{\prime}\left(r_{2}\right)^{5 / 3}\right], \\
F_{3}\left(r_{1}, r_{2}\right)= & \nu_{1}\left(r_{1}\right) \gamma_{1}\left(r_{2}\right)+x_{\alpha}\left[\left(\nu_{1}\left(r_{1}\right)+\nu_{1}\left(r_{2}\right)\right)^{4 / 3}-\nu_{1}\left(r_{1}\right)^{4 / 3}-\nu_{1}\left(r_{2}\right)^{4 / 3}\right]- \\
& -x_{k}\left[\left(\nu_{1}\left(r_{1}\right)+\nu_{1}\left(r_{2}\right)\right)^{5 / 3}-\nu_{1}\left(r_{1}\right)^{5 / 3}-\nu_{1}\left(r_{2}\right)^{5 / 3}\right], \\
F_{4}\left(r_{1}, r_{2}\right)=- & \nu_{1}^{\prime}\left(r_{1}\right) \gamma_{1}^{\prime}\left(r_{2}^{\prime}-x_{a}^{\prime}\left[\left(\nu_{1}^{\prime}\left(r_{1}\right)+\nu_{1}^{\prime}\left(r_{2}\right)\right)^{4 / 3}-\nu_{1}^{\prime}\left(r_{1}\right)^{4 / 3}-\nu_{1}^{\prime}\left(r_{2}\right)^{4 / 3}\right]+\right. \\
& +x_{k}\left[\left(\nu_{1}^{\prime}\left(r_{1}\right)+\nu_{1}^{\prime}\left(r_{2}\right)^{5 / 3}-\nu_{1}^{\prime}\left(r_{1}\right)^{5 / 3}-\nu_{1}^{\prime}\left(r_{2}\right)^{5 / 3}\right]-\right.
\end{aligned}
$$

According to Jensen, we introduce a set of new variables in the integrals :

$$
\begin{gathered}
r_{1}^{2}=x^{2}+y^{2}+z^{2}, \\
r_{2}^{2}=\left(\dot{\delta}_{0}-x\right)^{2}+y^{2}+z^{2}, \\
\operatorname{tg} \vartheta=\frac{z}{y} .
\end{gathered}
$$

The limits of integration are in the new variables for instance at the region (1)

$$
0 \leq \vartheta \leq 2 \pi, \quad \delta_{0}-r_{2} \leq r_{1} \leq r_{0}, \quad \delta_{0}-r_{01} \leq r_{2} \leq r_{02},
$$

and the volume element is

$$
d \tau=d x d y d z=\frac{\partial(x, y, z)}{\partial\left(r_{1}, r_{2}, \vartheta\right)} d r_{1} d r_{2} d \vartheta=\frac{r_{1} r_{2}}{\delta_{0}} d r_{1} d r_{2} d \vartheta
$$

The integration with respect to $\vartheta$ can be carried out immediately, since the model has a cylindric symmetry: 


$$
d \tau=\frac{2 \pi r_{1} r_{2}}{\delta_{0}} d r_{1} d r_{2}
$$

With two new variables we have again

$$
\begin{gathered}
\xi=r_{01}+r_{02}-r_{1}-r_{2}, \\
\eta=r_{01}-r_{02}-r_{1}+r_{2},
\end{gathered}
$$

and the new limits

$$
-\xi \leq \eta \leq \xi, \quad\left(i \leq \xi \leq r_{01}+r_{02}-\delta_{0},\right.
$$

and the volume element

$$
d \tau=\frac{2 \pi r_{1} r_{2}}{\delta_{0}} d r_{1} d r_{2}=\frac{2 \pi r_{1} r_{2}}{\delta_{0}} \frac{\partial\left(r_{1}, r_{2}\right)}{\partial(\xi, \eta)} d \xi d \eta=-\frac{\pi r_{1} r_{2}}{\delta_{0}} d \xi d \eta
$$

The integrals of (34) and (35) are now

$$
\begin{aligned}
& J_{1}=\int_{(1)} F_{1}\left(r_{1}, r_{2}\right) d \tau=\frac{\pi}{\delta_{0}} \int_{0}^{r_{01}+r_{01}-\delta 0} d \xi \int_{-\xi}^{\xi}\left[r_{1} r_{2} F_{1}\left(r_{1}, r_{2}\right)\right]_{r_{1}=r_{01}-\frac{\xi+\eta}{2}} d \eta
\end{aligned}
$$

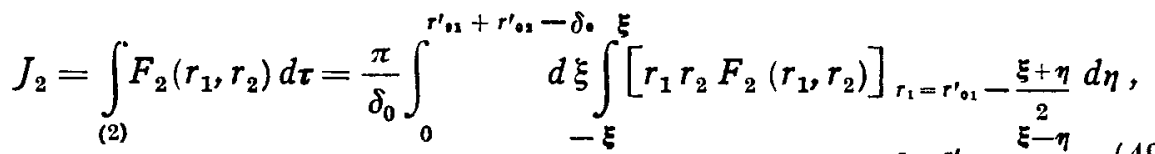

$$
\begin{aligned}
& r_{1}=r_{02}-\frac{\xi-\eta}{2} \\
& J_{3}=\int_{(3)} F_{3}\left(r_{1}, r_{2}\right) d \tau=\frac{\pi}{\sqrt{2} \delta_{0}} \int_{0}^{2 r_{01}-\sqrt{2} \delta_{0}} d \xi \int_{-\xi}^{\xi}\left[r_{1} r_{2} F_{3}\left(r_{1}, r_{2}\right)\right] r_{r_{1}=r_{01}-\frac{\xi+\eta}{2}} d \eta \\
& \left.J_{4}=\int_{(4)} F_{4}{ }^{\prime} r_{1}, r_{2}\right) d \tau=\frac{\pi}{\sqrt{2} \delta_{0}} \int_{0}^{2 r_{02}-y^{2} \overline{2} \delta_{0}} d \xi \int_{-\xi}^{\xi}\left[r_{1} r_{2} F_{4}\left(r_{1}, r_{2}\right)\right]_{r_{1}=r_{01}^{\prime}-\frac{\xi+\eta}{2}} d \eta
\end{aligned}
$$


The first and second derivatives of (48) to (51) with respect to $\delta$ are at $\delta=\delta_{0}$, (which are here the same as the derivatives with respect to $\delta_{0}$ ),

$$
\begin{aligned}
& \frac{d J_{1}}{d \delta_{0}}=-\frac{J_{1}}{\delta_{0}}-\frac{2 \pi}{\delta_{0}} \int_{\delta_{0}-r_{03}}^{r_{30}} r_{2}\left(\delta_{0}-r_{2}\right) F_{1}\left(\delta_{0}--r_{2}, r_{2}\right) d r_{2}, \\
& \frac{d J_{2}}{d \delta_{0}}=-\frac{J_{2}}{\delta_{\theta}}-\frac{2 \pi}{\delta_{0}} \int_{\delta_{0}-r_{01}}^{r^{\prime} r_{1}} r_{2}\left(\delta_{0}-r_{2}\right) F_{2}\left(\delta_{0}-r_{2}, r_{2}\right) d r_{2} \text {, } \\
& \frac{d J_{3}}{d \delta_{0}}=-\frac{J_{3}}{\delta_{0}}-\frac{2 \pi}{\delta_{0}} \int_{V_{2} \delta_{0}-r_{01}}^{r_{01}} r_{2}\left(\sqrt{2} \delta_{0}-r_{2}\right) F_{3}\left(\sqrt{2} \delta_{0}-r_{2}, r_{2}\right) d r_{2} \text {, } \\
& \frac{d J_{4}}{d \delta_{0}}=-\frac{J_{4}}{\delta_{0}}-\frac{2 \pi}{\delta_{0}} \int_{V_{2} \delta_{0}-r_{01}^{\prime \prime 2}}^{r_{1}} r_{2}\left(\sqrt{2} \delta_{0}-r_{2}\right) F_{4}\left(\sqrt{2} \delta_{0}-r_{2}, r_{2}\right) d r_{2}, \\
& \frac{d^{2} J_{J}}{d \delta_{0}^{2}}=-\frac{2}{\delta_{0}} \frac{d J_{1}}{d \delta_{0}}+\frac{2 \pi}{\delta_{0}}\left(\delta_{0}-r_{01}\right) r_{01} F_{1}\left(r_{01}, \delta_{0}-r_{01}\right)- \\
& -\frac{2 \pi}{\delta_{0}} \int_{\delta_{0}-r_{02}}^{r_{02}} r_{2}\left[F_{1}+r_{1} \frac{\partial F_{1}}{\partial r_{1}}\right]_{r_{1}-\delta_{0}-r_{1}} d r_{2} \\
& \frac{d^{2} J_{2}}{d \delta_{0}^{2}}=-\frac{2}{\delta_{0}} \frac{d J_{2}}{d \delta_{0}}+\frac{2 \pi}{\delta_{0}}\left(\delta_{0}-r_{01}^{\prime}\right) r_{01}^{\prime} F_{2}\left(r_{01}^{\prime}, \delta_{0}-r_{01}^{\prime}\right)- \\
& -\frac{2 \pi}{\delta_{0}} \int_{\delta_{0}-r_{02}^{\prime}}^{r^{\prime} r_{0}} r_{2}\left[F_{2}+r_{1} \frac{\partial F_{2}}{\partial r_{1}}\right]_{r_{1}=\delta_{0}-r_{1}} d r_{2} \\
& \frac{d^{2} J_{3}}{d \delta_{0}^{2}}=-\frac{2}{\delta_{0}} \frac{d J_{3}}{d \delta_{0}}+\frac{2 \sqrt{2} \pi}{\delta_{0}}\left(\sqrt{2} \delta_{0}-r_{01}\right) r_{01} F_{3}\left(r_{01}, \sqrt{2} \delta_{0}-r_{01}\right)- \\
& -\frac{2 \sqrt{2} \pi}{\delta_{0}} \int_{\sqrt{2} \delta_{0}-r_{01}}^{r_{01}} r_{2}\left[F_{3}+r_{1} \frac{\partial F_{3}}{\partial r_{1}}\right]_{r_{1}=\sqrt{2} \delta_{0}-r_{1}} d r_{2} \\
& \frac{d^{2} J_{4}}{d \delta_{0}^{2}}=-\frac{2}{\delta_{0}} \frac{d J_{4}}{d \delta_{0}}+\frac{2 \sqrt{2} \pi}{\delta_{0}}\left(\sqrt{2} \delta_{\mathrm{J}}-r_{01}^{\prime}\right) r_{01}^{\prime} F_{4}\left(r_{01}^{\prime}, \sqrt{2} \delta_{0}-r_{01}^{\prime}\right)-
\end{aligned}
$$




$$
-\frac{2 \sqrt{2} \pi}{\delta_{3}} \int_{\sqrt{2} \delta_{0}-r_{01}^{\prime}}^{r_{01}^{\prime}} r_{2}\left[F_{4}+r_{1} \frac{\partial F_{4}}{\partial r_{1}}\right]_{r_{1}=\sqrt{2} \delta_{0}-r_{3}} d r_{2} .
$$

The integrals occurring in (56) to (59) vanish in a good approximation. In the integral of (56), for example, we have approximately

$$
F_{1}\left(r_{1}, r_{2}\right)+r_{1} \frac{\partial F_{1}\left(r_{1}, r_{0}\right)}{\partial r_{1}}=F_{1}\left(2 r_{1}, r_{2}\right) \text {. }
$$

Since the relation $\delta_{0}-r_{02} \leq r_{1} \leq r_{01}$ holds in this integral, and since we have $r_{01}<2\left(\delta_{0}-r_{02}\right) \leq 2 r_{1}$ from the numerical data, (60) means by the definition (36) of $F_{1}$, that

$$
F_{1}\left(2 r_{1}, r_{2}\right)=0
$$

This and a similar treatment with the other integrals gives for (56) to (59)

$$
\begin{gathered}
\frac{d^{2} J_{1}}{d \delta_{0}^{2}}=-\frac{2}{\delta_{0}} \frac{d J_{1}}{d \delta_{0}}+\frac{2 \pi}{\delta_{0}} r_{01}\left(\delta_{0}-r_{01}\right) F_{1}\left(r_{01}, \delta_{0}-r_{01}\right), \\
\frac{d^{2} J_{2}}{d \delta_{0}^{2}}=-\frac{2}{\delta_{0}} \frac{d J_{2}}{d \delta_{0}}+\frac{2 \pi}{\delta_{0}} r_{01}^{\prime}\left(\delta_{0}-r_{01}^{\prime}\right) F_{2}\left(r_{01}^{\prime}, \delta_{0}-r_{01}^{\prime}\right), \\
\frac{d^{2} J_{3}}{d \delta_{0}^{2}}=-\frac{2}{\delta_{0}} \frac{d J_{3}}{d \delta_{0}}+\frac{2 \sqrt{2} \pi}{\delta_{0}} r_{01}\left(\sqrt{2} \delta_{0}-\cdots r_{01}\right) F_{3}\left(r_{01}, \sqrt{2} \delta_{0}-r_{01}\right), \\
\frac{d^{2} J_{4}}{d \delta_{0}^{2}}=-\frac{2}{\delta_{0}} \frac{d J_{4}}{d \delta_{0}}+\frac{2 \sqrt{2} \pi}{\delta_{0}} r_{01}^{\prime}\left(\sqrt{2} \delta_{0}-r_{01}^{\prime}\right) F_{4}\left(r_{01}^{\prime}, \sqrt{2} \delta_{0} \cdots r_{01}^{\prime}\right)
\end{gathered}
$$

In the integrals (52) to (55) there occur terms of the form $\left(\nu_{1}\left(r_{1}\right)+\right.$ $\left.+\nu_{2}\left(r_{2}\right)\right)^{\alpha}$ or $\left(\nu_{1}\left(r_{1}\right)+\nu_{1}\left(r_{2}\right)\right)^{\alpha}$. These can be simplified by the following method. In (52), for instance, the density of $R b^{+}$is very large against that of $\mathrm{I}^{-}$in nearly $82 \%$ of the region of integration. (See figure 3.) In these parts we have

$$
\begin{aligned}
\left(\nu_{1}\left(r_{1}\right)+\nu_{2}\left(r_{2}\right)\right)^{\alpha} & =\nu_{2}\left(r_{2}\right)^{\alpha}\left(1+\frac{\nu_{1}\left(r_{1}\right)}{\nu_{2}\left(r_{2}\right)}\right)^{\alpha}=\nu_{2}\left(r_{2}\right)^{\alpha}+ \\
& +\alpha \nu_{1}\left(r_{1}\right) \nu_{2}\left(r_{2}\right)^{\alpha-1}
\end{aligned}
$$




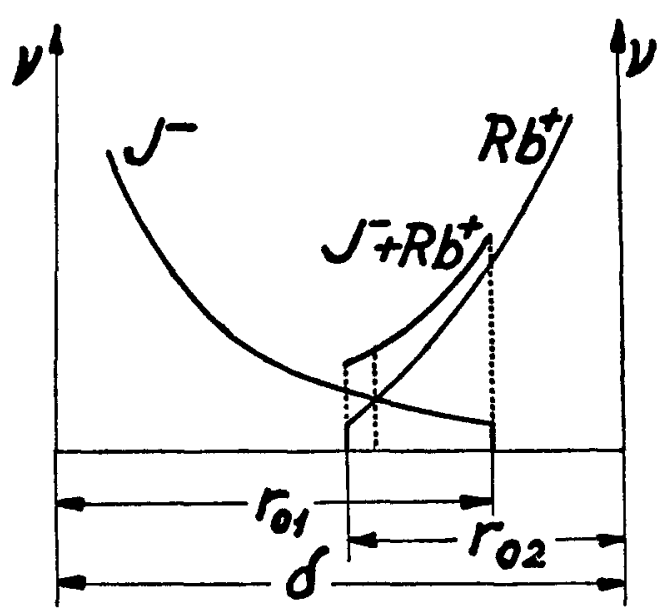

Fig. 3.

If we keep valid this expression for the remaining $18 \%$ also, we do not make a great mistake regarding the smallness of the region of integration and regarding that the largest amount of the difference from the exact value is less than $12 \%$ for $\alpha=4 / 3$ and less than $26 \%$ for $\alpha=5 / 3$. So we can use in the integral of (52) the expansion (66).

In (53) we have even a more convenient feature. The regions of integration are here still smaller. Here we have to make an expansion in $\Delta \nu / \nu$ also, which is allowed always since $\Delta \nu \ll \nu$.

In (54) and (55) we cut the region of integration into two equal parts at $\delta_{0} / \sqrt{2}$; the integrals in each part are the same and so we can take twice the integral in one part. But in one part one $\nu$ is always larger than the other and so the expansions are valid.

So we have finally the following numerical results:

$$
\begin{aligned}
& \frac{d J_{1}}{d \delta_{0}}=-\frac{J_{1}}{\delta_{0}}+0,00855, \\
& \frac{d J_{2}}{d \delta_{0}}=-\frac{J_{2}}{\delta_{0}}-0,00048, \\
& \frac{d J_{3}}{d \delta_{0}}=-\frac{J_{3}}{\delta_{0}}-0,00588, \\
& \frac{d J_{4}}{d \delta_{0}}=-\frac{J_{4}}{\delta_{0}}-0,00004,
\end{aligned}
$$




$$
\begin{aligned}
& \frac{d^{2} J_{1}}{d \delta_{0}^{2}}=-\frac{2}{\delta_{0}} \frac{d J_{1}}{d \delta_{0}}-0,00287=\frac{2 J_{1}}{\delta_{0}^{2}}-0,00515 \\
& \frac{d^{2} J_{2}}{d \delta_{0}^{2}}=-\frac{2}{\delta_{0}} \frac{d J_{2}}{d \delta_{0}}+0,00565=\frac{2 J_{9}}{\delta_{0}^{2}}+0,00579 \\
& \frac{d^{2} J_{3}}{d \delta_{0}^{2}}=-\frac{2}{\delta_{0}} \frac{d J_{3}}{d \delta_{0}}-0,00034=\frac{2 J_{3}}{\delta_{0}^{2}}+0,00123 \\
& \frac{d^{2} J_{4}}{d \delta_{0}^{2}}=-\frac{2}{\delta_{0}} \frac{d J_{4}}{d \delta_{0}}+0,00084=\frac{2 J_{4}}{\delta_{0}^{2}}+0,00085
\end{aligned}
$$

From (33), (23), (34), (35), (67)-(74) we have now

$$
\Delta \delta_{\nu}=-\frac{\frac{\Delta E}{\delta_{0}}+0,01290}{\left[\frac{d^{2} E}{d \delta^{2}}\right]_{\delta=\delta_{0}}+\frac{2 \Delta E}{\delta_{0}^{2}}+0,01632}
$$

cit.). Namely

$\left[\frac{d^{2} E}{d \delta^{2}}\right]_{\delta=\delta_{0}}$ can be taken from Jensen's compressibility (see loc.

$$
\left\lceil\frac{d^{2} E}{d \delta^{2}}\right]_{\delta=\delta_{0}}=\frac{18 \delta_{0}}{x}
$$

$x$ meaning the compressibility. Jensen gives $x=15,5 \cdot 10^{-12} \mathrm{~g}^{-1} \mathrm{~cm} \sec ^{2}$ and so

$$
\left[\frac{d^{2} E}{d \delta^{2}}\right]_{\delta=\delta_{0}}=0,02948
$$

Estimations show that $\Delta E / \delta_{0}$ and $2 \Delta E / \delta_{0}^{2}$ give a contribution of only about $10 \%$ and $2 \%$ respectively to the numerically evaluated terms of (75). This means that, if we neglect these terms, we make a mistake of $12 \%$ in $\Delta \delta_{0}, \mathrm{i}, \mathrm{e}$, a mistake of $0,4 \%$ in $\delta_{0}^{\prime}$, enlarging $\Delta \delta_{0}$. For $\Delta \delta_{0}$ we have thus finally

$$
\Delta \delta_{0}=-(0,28+0,03)=-0,31
$$

i. e. for the lattice constant 


$$
\delta_{0}^{\prime}=7,17
$$

This value has a deviation of only $3,4 \%$ from the experimental value. Now if we take into account the van der Waals correction also, we have to use in the first approximation only Jensen's $x$, calculated with the van der Waals forces, $x=13,0 \cdot 10^{-12} \mathrm{~g}^{-1} \mathrm{~cm} \mathrm{sec} \mathrm{se}^{2}$. This gives

and so

$$
\left[\frac{d^{2} E}{d \delta^{2}}\right]_{\delta=\delta_{0}}=0,03407
$$

and

$$
\Delta \delta_{0}=-\cdots, 28
$$

$$
\delta_{0}^{\prime}=6,97
$$

giving only $0,6 \%$ deviation from the experimental data. Naturally the same note is valid here as in Jensen's work according to the use of the van der Waals forces.

The correction of the lattice energy results chiefly from that of $\delta_{0}$ in the Coulomb term. This gives a correction of nearly $4 \%$ to Jensen's lattice energy. The exact computation of the energy would mean the evaluation of two further double integrals.

\section{4. §. THE COMPRESSIBILITY AT ZERO ABSOLUTE TEMPERATURE.}

Using the preceeding method we can calculate other important properties of the $R b I$ crystal too. The definition of the compressibility being, for instance,

$$
x^{\prime}=-\frac{1}{V} \frac{d V}{d P},
$$

where $V$ is the volume per ion pair, and since at zero absolute temperature

$$
P=-\frac{d E^{\prime}}{d V},
$$

we have

$$
\frac{1}{\varkappa^{\prime}}=-V \frac{d P}{d V}=V \frac{d^{2} E^{\prime}}{d V^{2}}
$$

In our case of a face-centred cubic lattice there is 


$$
V=2 \delta^{3}
$$

and so

$$
\frac{1}{\varkappa^{\prime}}=\frac{1}{18 \delta}\left(-\frac{2}{\delta} \frac{d E^{\prime}}{d \delta}+\frac{d^{2} E^{\prime}}{d \delta^{2}}\right) .
$$

In the equilibrium state we have by $(30)$

$$
\frac{1}{\varkappa^{\prime}}=\frac{1}{18 \delta_{0}^{\prime}}\left[\frac{d^{2} E^{\prime}}{d \delta^{2}}\right]_{\delta=\delta_{0}^{\prime}}
$$

Here we can be content by taking the new value for $\delta_{0}^{\prime}$, where it occurs explicitly, and substituting the old $\delta=\delta_{0}$ instead of $\delta=\delta_{0}^{\prime}$ in the derivatives. Thus

$$
\frac{1}{x^{\prime}}=\frac{1}{18 \delta_{0}^{\prime}}\left[\frac{d^{2} E}{d \delta^{2}}\right]_{\delta=\delta_{0}}+\frac{1}{18 \delta_{0}^{\prime}}\left[\frac{d^{2} \Delta E}{d \delta^{2}}\right]_{\delta=\delta_{0}},
$$

i. e. we have the following values:

$$
x^{\prime}=: 9,56 \cdot 10^{-12} \mathrm{~g}^{-1} \mathrm{~cm} \mathrm{sec}{ }^{2}
$$

without the van der Waals forces and

$$
\varkappa^{\prime}=8,45 \cdot 10^{-12} \mathrm{~g}^{-1} \mathrm{~cm} \mathrm{sec}^{2}
$$

with the van der Waals forces.

These results cannot be compared directly with the experimental value because these are referring to the zero absolute temperature, while the experimental one refers to room temperature or higher temperatures. So the real value lies between that for $T=273^{\circ}$ and that linearly extrapolated to $T=0^{\circ}$.

$$
\begin{aligned}
& \varkappa_{273^{\circ}}=9,4 \cdot 10^{-12} \mathrm{~g}^{-1} \mathrm{~cm} \mathrm{sec}^{2}, \\
& \varkappa_{0^{\circ}}=7,6 \cdot 10^{-12} \mathrm{~g}^{-1} \mathrm{~cm} \mathrm{sec}^{2} .
\end{aligned}
$$

As we see, the $\varkappa^{\prime}$ calculated with the van der Waals forces lies exactly in the middle of the two boundaries.

\section{5. §. THE INFRA-RED WAVE-LENGTH.}

We can calculate the infra-red frequency of the crystal too. The intra-red vibration of the alkali halogenide ionic lattices consists of the relative vibration of two lattices. We shall consider namely 
the lattices consisting of the same kind of ions as rigid and let make little oscillations of the two lattices about the equilibrium state in the sense marked by the arrows in figure 4 .

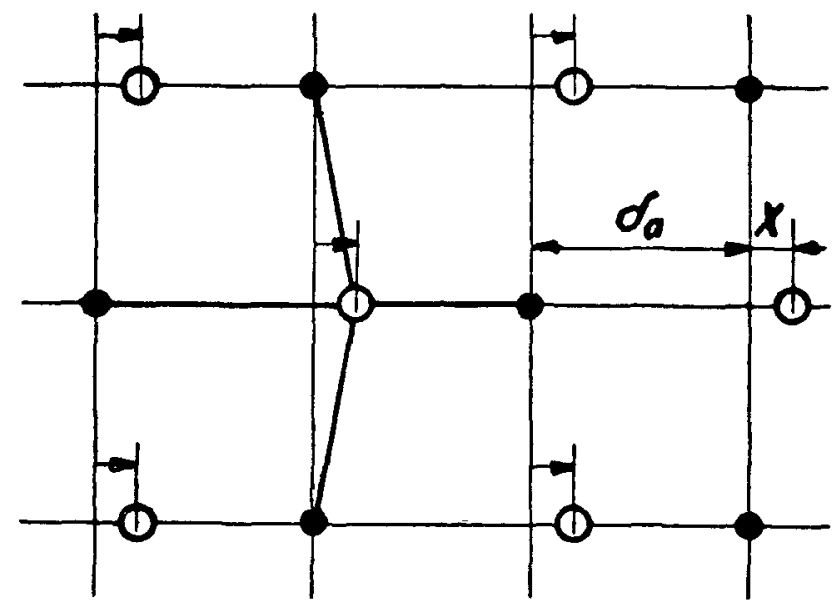

Fig. 4.

Denoting the elongation from the equilibrium position by $x$ and the whole lattice energy per ion pair by $U^{\prime}(x)$, we have in the equilibrium state

$$
\left[\frac{d U^{\prime}(x)}{d x}\right]_{x=0}=0
$$

and at a point $x$ near $x=0$

$$
-\frac{d U^{\prime} x}{d x}=-\left[\frac{d U^{\prime}}{d x}\right]_{x=0}-x\left[\frac{d^{2} U^{\prime}}{d x^{2}}\right]_{x=0}=-x\left[\frac{d^{2} U^{\prime}}{d x^{2}}\right]_{x=0} .
$$

This force acts on an ion pair. If we take the centre of mass of the two ions as fixed, we have to introduce the reduced mass by

$$
\frac{1}{M}=\frac{1}{M_{1}}+\frac{1}{M_{2}}
$$

$M_{1}$ and $M_{2}$ being the masses of the two ions respectively. So the equation of motion becomes

$$
M \ddot{x}=-x\left[\frac{d^{2} U^{\prime}}{d x^{2}}\right]_{x=0},
$$


i. $\varepsilon$.

$$
\ddot{x}+\left(\frac{1}{M_{1}}+\frac{1}{M_{2}}\right)\left[\frac{d^{2} U^{\prime}}{d x^{2}}\right]_{x=0} x=0 \text {. }
$$

This means a vibration with the frequency $\omega^{\prime}$, where

$$
()^{\prime 2}=\left(\frac{1}{M_{1}}+\frac{1}{M_{2}}\right)\left[\frac{d^{2} U^{\prime}}{d x^{2}}\right]_{x=0} \text {. }
$$

As we take the lattice of the ions of the same kind as rigid, the derivatives of the interaction terms of such ions with respect to $x$ vanish. So we have to take into the account only the interaction between the ionic charges and the interaction resulting from the overlapping of ions of opposite signs.

$$
\begin{gathered}
{\left[\frac{d^{2} U^{\prime}}{d x^{2}}\right]_{x=0}=\left[\frac{d^{2} U_{0}^{\prime}}{d x^{2}}\right]_{x=0}+\left[\frac{d^{2} U_{1}^{\prime}}{d x^{2}}\right]_{x=0},} \\
{\left[\frac{d^{2} U_{0}^{\prime}}{d x^{2}}\right]_{x=0}=-\frac{2 \pi}{3 \delta_{0}^{\prime 3}}=\left[\frac{d^{2} U_{0}}{d x^{2}}\right]_{x=0}+\frac{2 \pi d \delta_{0}}{\delta_{0}^{4}},}
\end{gathered}
$$

denoting Jensen's quantities without comma. As we can see in figure 4, the distances from the right and left neighbours are

$$
\delta=\delta_{0}^{\prime}-x \text { and } \delta=\delta_{0}^{\prime}+x
$$

respectively and from the other four neighbours

So we have

$$
\delta=\sqrt{\delta_{0}^{\prime 2}+x^{2}}==\delta_{0}^{\prime}+\frac{x^{2}}{2 \delta_{0}^{\prime}}
$$

$$
U_{1}^{\prime} x=\frac{1}{6} E_{1}^{\prime}\left(\delta_{0}^{\prime}-x\right)+\frac{1}{6} E_{1}^{\prime}\left(\delta_{0}^{\prime}+x\right)+\frac{2}{3} E_{1}^{\prime}\left(\delta_{0}^{\prime}+\frac{x^{2}}{2 \delta_{0}^{\prime}}\right)
$$

Differentiating twice with respect to $x$ and taking $x=0$ :

$$
\left[\frac{d^{2} U_{1}^{\prime}}{d x^{2}}\right]_{x=0}=\frac{1}{3}\left[\frac{d^{2} E_{1}^{\prime}}{d \delta^{2}}\right]_{\delta=\delta_{0}^{\prime}}+\frac{2}{3 \delta_{0}^{\prime}}\left[\frac{d E_{1}^{\prime}}{d \delta}\right]_{\delta=\delta_{0}^{\prime}} .
$$

Substituting $\delta_{0}$ instead of $\delta_{0}^{\prime}$ in the derivatives, 
$\left[\frac{d^{2} U_{1}^{\prime}}{d x^{2}}\right]_{x=0}=\left[\frac{d^{2} U_{1}}{d x^{2}}\right]_{x=0}+\frac{1}{3}\left[\frac{d^{2} \Delta E}{d \delta^{2}}\right]_{\delta=\delta_{0}}+\frac{2}{3 \delta_{0}^{\prime}}\left[\frac{d \Delta E}{d \delta}\right]_{\delta=\delta_{0}}$

and finally

$$
\begin{gathered}
{\left[\frac{d^{2} U^{\prime}}{d x^{2}}\right]_{x=0}=\left[\frac{d^{2} U}{d x^{2}}\right]_{x=0}+\frac{2 \pi \Delta \delta_{1}}{\delta_{0}^{4}}+} \\
+\frac{1}{3}\left[\frac{d^{2} \Delta E}{d \delta^{2}}\right]_{\delta=\delta_{0}}+\frac{2}{3 \delta_{0}^{\prime}}\left[\frac{d \Delta E}{d \delta}\right]_{\delta=\delta_{0}}=\left[\frac{d^{2} U}{d x^{2}}\right]_{x=0}+\varepsilon,
\end{gathered}
$$

which serves at the same time as the definition of $\varepsilon$. So

$$
\begin{gathered}
\omega^{\prime 2}=\left(\frac{1}{M_{1}}+\frac{1}{M_{2}}\right)\left[\frac{d^{2} U}{d x^{2}}\right]_{x=0}+\left(\frac{1}{M_{1}}+\frac{1}{M_{2}}\right) \varepsilon=\omega^{2}+ \\
\left(\frac{1}{M_{1}}+\frac{1}{M_{2}}\right) \varepsilon .
\end{gathered}
$$

On the other hand we have

$$
\omega^{\prime 2}=\left(\omega+\Delta \omega^{2}=\omega^{2}+2 \omega \Delta \omega\right.
$$

and so from (96) and (97):

$$
\frac{\Delta \omega}{\omega}=\left(\frac{1}{M_{1}}+\frac{1}{M_{2}}\right) \frac{\varepsilon}{2 \omega^{2}}=\left(\frac{1}{M_{1}}+\frac{1}{M_{2}}\right) \frac{\lambda^{2} \varepsilon}{8 \pi^{2} c^{2}}
$$

and

$$
\begin{gathered}
\lambda^{\prime}=\frac{2 \pi c}{\omega^{\prime}}=\frac{2 \pi c}{\omega}- \\
-\frac{2 \pi c \Delta \omega}{\omega^{2}}=\lambda\left(1-\frac{\Delta \omega}{\omega}\right)=\lambda-\frac{1}{8 \pi^{2} c^{2}}\left(\frac{1}{M_{1}}+\frac{1}{M_{2}}\right) \lambda^{3} \varepsilon .
\end{gathered}
$$

So we have instead of Jensen's

$$
\lambda=215 \cdot 10^{-4} \mathrm{~cm} \text { and } \lambda==195 \cdot 10^{-4} \mathrm{~cm}
$$

without and with the van der Waals correction respectively

$$
\lambda^{\prime}=82 \cdot 10^{-4} \mathrm{~cm} \text { and } \lambda^{\prime}=24 \cdot 10^{-4} \mathrm{~cm}
$$


the experimental value being

$$
\lambda_{\exp }=29 \cdot 10^{-4} \mathrm{~cm} \text {. }
$$

We can make a rough approximation of the mechanical strength too, but the results cannot be compared with the experimental value, since the theoretical and experimental circumstances are very different.

\section{6. §. SUMMARY.}

The table below shows Jensen's results and those, which I have got, together with their percentual deviation from the experimental values. ${ }^{16}$

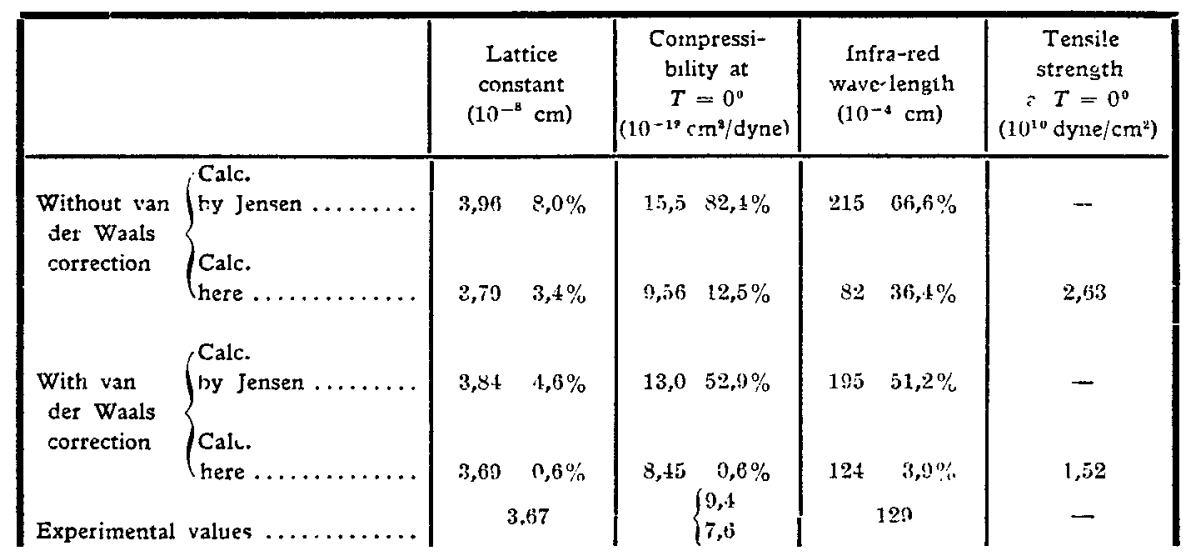

As we see, Jensen's calculations with the Fermi-Amaldi model show fairly large percentual deviations from the experimental results. This is caused by the unsatisfactory description of the outer parts of the ions in the model used. In the Gombás model we take into account the correlation effect also, which improves the results extremely well, so that we may conclude, that in a similar treatment of the alkali halogenides there is no need of a further improvement of the statistical model.

The present work was performed in the Physical Institute of the Hungarian University for Technical and Economic Sciences. I wish to express my thanks to Prof. P. Gombás for his kind encouraging to solve this problem and for his valuable advises.

16 The table of results given in Phys. Rev, 70, p. 981 (1946) by the author is somewhat corrected here, further see footnote 15. 